EPJ Web of Conferences 113,01012 (2016)

DOI: 10.1051/epjconf/201611301012

(C) Owned by the authors, published by EDP Sciences, 2016

\title{
Experimental progress and status on nucleon electromagnetic form factors
}

\author{
H. Gao ${ }^{1,2, a}$, C. Peng ${ }^{1, b}$, and Z.W. Zhao ${ }^{1, c}$ \\ ${ }^{1}$ Duke University, Durham, NC27708, U.S.A. \\ ${ }^{2}$ Duke Kunshan University, Kunshan, Jiangsu, China 215316
}

\begin{abstract}
Nucleon electromagnetic form factors are the fundamental quantities that are related to a two-dimensional view of the charge and magnetization distribution in the nucleon. They are essential in understanding the nucleon electromagnetic structure. In this article, we briefly summarize the recent experimental progress on this topic, and introduce several experiments related to the nucleon electromagnetic form factors in the near future.
\end{abstract}

\section{Introduction}

Nucleons are known as the fundamental building blocks of the visible matter. In 1933, Otto Stern discovered that the magnetic momentum of proton disagreed with Dirac's prediction $\mu=\frac{e}{2 M} \bar{h}$ for a structureless spin- $\frac{1}{2}$ particle. The discovery firstly indicated the internal structure of nucleons. In 1950s, the electron-proton elastic scattering experiments by Hofstadter and others confirmed the spatial charge distribution of the nucleon. A decade later, the experimental efforts on deep-inelastic scattering of electrons on protons pioneered by Friedman, Kendall and Taylor at Stanford Linear Accelerator Center (SLAC) revealed the point-like constituent particles inside the proton, and led to the establishment of the quark model.

Quantum Chromodynamics (QCD) is the fundamental theory that describes the strong interaction between quarks and gluons. Although it was well tested in the high energy region, where the quarks are asymptotically free and perturbative QCD is applicable, the predictions and tests regarding the confinement and hadron structure in the non-perturbative regime are extremely challenging. Thus, the experimental efforts in revealing the internal structure of nucleons are essential for understanding QCD and the fundamental interactions at the level of quarks and gluons.

Among fundamental quantities related to the internal structure of the nucleon, the electromagnetic form factors describe the spatial distribution of charge and magnetization within the nucleon. The recent measurements of nucleon form factors at high $Q^{2}$ inspired many topics regarding the nucleon structure: the possible relations between $G_{E}^{p} / G_{M}^{p}$ and the quark orbital angular momentum [1], the behaviors of individual quark-flavor contributions to the nucleon form factors [2], and the role of form factors in the unified view of nucleon structure based on the Wigner distributions. Besides, the

\footnotetext{
ae-mail: gao@phy.duke.edu

be-mail: cp121@phy.duke.edu

ce-mail: zwzhao@jlab.org
} 
so-called "proton charge radius puzzle" [3] has raised many interests to re-evaluate the proton charge radius value extracted from the electric form factor of the proton at low $Q^{2}$ region and to carry out new experiments to access previously unexplored lower $Q^{2}$ region.

The rest of this paper will introduce the experimental techniques and the recent experimental progress related to the nucleon form factors, as well as provide a perspective on the future experiments regarding this topic. This proceedings paper focuses on nucleon form factors in the space-like region only.

\section{Experimental techniques}

\subsection{Rosenbluth separation}

In the one-photon exchange approximation, the differential cross section of unpolarized elastic electron-proton scattering can be written as the Rosenbluth formula [4]:

$$
\frac{d \sigma}{d \Omega}=\frac{\sigma_{M}}{\epsilon} \frac{\tau}{1+\tau}\left(\frac{\epsilon}{\tau} G_{E}^{p 2}+G_{M}^{p 2}\right), \text { and } \sigma_{\mathrm{M}}=\frac{\alpha \mathrm{E}^{\prime} \cos ^{2} \frac{\theta}{2}}{4 \mathrm{E}^{3} \sin ^{4} \frac{\theta}{2}},
$$

,where $\tau=Q^{2} /\left(4 M_{p}^{2}\right), \epsilon=\left(1+2(1+\tau) \tan ^{2} \frac{\theta}{2}\right)^{-1}$ is the virtual photon longitudinal polarization, and $\sigma_{M}$ is the Mott cross section describing the scattering from a point-like target.

In Rosenbluth method, the proton electric and magnetic form factor squared $G_{E}^{p 2}$ and $G_{M}^{p 2}$ are separated by measuring the cross-section with varied beam energies and electron scattering angles but fixed $Q^{2}$. The reduced cross section $\sigma_{R}=\frac{\epsilon}{\tau} G_{E}^{p}{ }^{2}+G_{M}^{p 2}$ at fixed $Q^{2}$ only depends on $\epsilon$ (or the scattering angle $\theta) . G_{E}^{p}{ }^{2}$ and $G_{M}^{p 2}$ hence can be extracted as the slope and the intercept of $\sigma_{R}(\epsilon)$, respectively. Since the contribution from $G_{M}$ term dominates the cross-section in the high $Q^{2}$ region, while $G_{E}$ term becomes dominant as the $Q^{2}$ decreases, it is difficult to extract the $G_{E}$ at large $Q^{2}$ and the $G_{M}$ at low $Q^{2}$ using Rosenbluth method.

\subsection{Recoil proton polarization measurement}

Since the elastic scattering cross-section is much less sensitive to the electric form factor at high $Q^{2}$, the relative uncertainties of the $G_{E}^{p}$ data determined by the Rosenbluth method increases drastically at $Q^{2}>1 \mathrm{GeV}^{2}$. Nevertheless, experimental techniques using the polarization degrees of freedom in electron scattering provide an access to the interference term of the form factor $G_{E} G_{M}$, which does not suffer from the $Q^{2}$ dependent sensitivity. As one of such methods, the experiment based on the elastic scattering of longitudinally polarized electrons from unpolarized protons can obtain the ratio of form factors $G_{E} / G_{M}$ by simultaneously measuring the transverse $\left(P_{l}\right)$ and longitudinal $\left(P_{t}\right)$ polarizations of the recoil proton:

$$
\frac{G_{E}^{p}}{G_{M}^{p}}=-\frac{P_{t}}{P_{l}} \frac{E+E^{\prime}}{2 M} \tan \frac{\theta}{2}
$$

The recoil proton polarizations are extracted from the azimuthal distribution after recoil protons scattering on certain analyzer material, which is typically carbon. The experimental observable from recoil proton polarization measurement directly determines the ratio of form factors. It does not require several measurements at the same $Q^{2}$, and thus significantly reduces the systematical errors regarding the change of the kinematic settings. This method cancels many factors that would introduce experimental uncertainties, such as the beam polarization and luminosity, the detector efficiency, 
and the analyzing power of proton polarimeter. Combining cross-section measurements and recoil proton polarization measurements, one can precisely determine both form factors regardless of their sensitivities to $Q^{2}$.

\subsection{Asymmetry super ratio method}

Another method to extract the ratio of proton form factors is to measure the asymmetry from the elastic scattering of longitudinally polarized electrons from polarized protons. The experimental asymmetry can be written as:

$$
A_{\exp }=P_{b} P_{t} \frac{-2 \tau v_{T^{\prime}} \cos \theta^{*} \mathrm{G}_{\mathrm{M}}^{\mathrm{p} 2}+2 \sqrt{2 \tau(1+\tau)} v_{\mathrm{TL}} \sin \theta^{*} \cos \phi \mathrm{G}_{\mathrm{M}}^{\mathrm{p}} \mathrm{G}_{\mathrm{E}}^{\mathrm{p}}}{(1+\tau) v_{L} G_{E}^{p 2}+2 \tau v_{T} G_{M}^{p 2}},
$$

where $\theta^{*}$ and $\phi^{*}$ are the polar and azimuthal angles of the proton polarization with respect to the three-momentum transfer of the virtual photon and the scattering plane, $P_{b}$ and $P_{t}$ are beam and target polarizations, respectively, $v_{T^{\prime}}, v_{T L^{\prime}}, v_{T}$ and $v_{L}$ are kinematic factors [5]. The ratio of proton form factors is thus determined by the "super ratio" when one uses a symmetric detector system [6]:

$$
R_{A}=\frac{A_{1}}{A_{2}}=\frac{a_{1}-b_{1} G_{E}^{p} / G_{M}^{p}}{a_{2}-b_{2} G_{E}^{p} / G_{M}^{p}} .
$$

$A_{1}$ and $A_{2}$ are the measured asymmetries in the two symmetric detectors with the proton polarization fixed in the laboratory frame. This technique also benefits from the cancellation of detector efficiency, beam and target polarizations, and luminosity. Its systematic uncertainties are different with those of the recoil polarization measurements.

\subsection{Neutron form factor measurement}

The measurement of neutron form factors is challenging due to the lack of free neutron targets. Therefore, unpolarized elastic or quasi-elastic electron-deuteron scattering, coupled with theoretical deuteron calculations, is employed to extract the neutron form factors. However, a strong model dependence is involved with this technique, and the precision is worse in comparison to the proton form factors measurements.

The direct measurement of neutron electric form factor $G_{E}^{n}$ is even more difficult from the unpolarized cross-section measurements, due to the fact that the contribution from magnetic form factor $G_{M}^{n}$ dominates the cross-sections. Alternatively, the ratio of neutron form factor is extracted from the polarization observables. The experimental technique can be either achieved by measuring the recoil neutron polarization from the process $d\left(\vec{e}, e^{\prime} \vec{n}\right)$, or by measuring the spin-dependent asymmetry from the process $\vec{d}\left(\vec{e}, e^{\prime} n\right)$.

An alternative target for the spin-dependent quasi-elastic scattering measurements is polarized ${ }^{3} \mathrm{He}$. As an effective neutron target, the ground state of ${ }^{3} \mathrm{He}$ is dominated by the $\mathrm{S}$ wave, in which the spins of two protons cancel out, and the unpaired neutron carries the ${ }^{3} \mathrm{He}$ nuclear spin. The polarized ${ }^{3} \mathrm{He}$ target is typically utilized in two types of experiments: inclusive ${ }^{3} \mathrm{He}\left(\vec{e}, e^{\prime}\right)$ measurement in which only the scattered electron is detected, and coincidence ${ }^{3} \mathrm{He}\left(\vec{e}, e^{\prime} n\right)$ measurement in which both the scattered electron and knockout neutron are detected to minimize the asymmetry contribution from protons. 

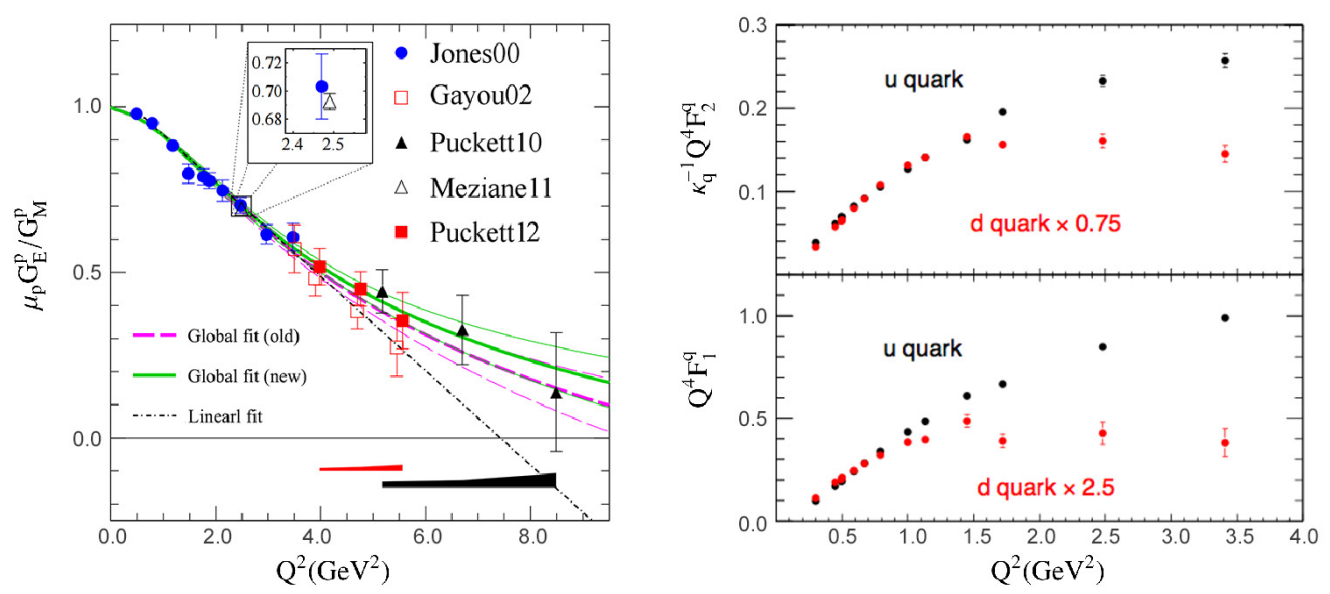

Figure 1. The left plot is the proton electromagnetic form factor ratio $\mu_{p} G_{E}^{p} / G_{M}^{p}$ from [9]. The right plot is the discomposed up and down quark contributions to the proton form factors from [2].

\section{Progress on nucleon form factors}

\subsection{Relation to quark orbital angular momentum}

The prediction of nucleon form factors at large $Q^{2}$ values was given by Brodsky and Farrar [7] based on the dimensional counting rules, implying the Dirac form factor $F_{1}^{p} \propto Q^{-4}$, and the Pauli form factor $F_{2}^{p}$ has an additional factor of $Q^{-2}$ due to helicity conservation. Thus, $Q^{2} F_{2}^{p} / F_{1}^{p}$ (or $G_{E}^{p} / G_{M}^{p}$ ) should become constant at sufficiently large $Q^{2}$. This prediction was then confirmed with the perturbative QCD analysis given by Brodsky and Lepage [8]. However, as shown in Fig 1 (left), the current experimental data of the electromagnetic form factors ratio $G_{E}^{p} / G_{M}^{p}$ decrease as $Q^{2}$ gets larger.

The scaling factor was then revisited by the pQCD analysis of nucleon's Pauli form factor [10] considering both leading- and subleading-twist light-cone wave functions, the latter describes quarks with one unit of orbital angular momentum. Its result predicts that $F_{2}^{p} / F_{1}^{p} \sim \ln ^{2}\left(\mathrm{Q}^{2} / \Lambda^{2}\right) / \mathrm{Q}^{2}$, where $\Lambda$ is a soft scale parameter related to the nucleon size. With a selection of $\Lambda=0.2-0.3 \mathrm{GeV}^{2}$, this prediction has a good agreement with the experimental data at $Q^{2}>1 \mathrm{GeV}^{2}$.

Many relativistic constituent quark models (rCQM) [11-16] predict that $G_{E}^{p} / G_{M}^{p}$ decreases with the increase of $Q^{2}$. This is related to the fact that these models, by imposing Poincare invariance, lead to substantial violation of hadron helicity conservation, i.e., they include non-zero quark orbital angular momentum. A calculation based on Dyson Schwinger Equation (DSE) and Faddeev equation from Argonne [17] also predicts the decreasing behavior of $G_{E}^{p} / G_{M}^{p}$ over $Q^{2}$. The calculation involves many interesting features, such as the dynamically generated quark mass, the contribution of quark orbital angular momentum, and the incorporation of diquark degrees of freedom.

\subsection{Flavor decomposition of nucleon form factors}

With experimental data on both $G_{E}^{p}$ and $G_{E}^{n}$, the contributions from individual quark flavor can be extracted by assuming the charge asymmetry. Such a flavor decomposition was recently carried out for $Q^{2}$ values up to $3.4 \mathrm{GeV}^{2}$ [2]. The relations between nucleon form factors and the up or down quark contributions are: 


$$
F_{1(2)}^{u}=2 F_{1(2)}^{p}+F_{1(2)}^{n} \text {, and } \mathrm{F}_{1(2)}^{\mathrm{d}}=2 \mathrm{~F}_{1(2)}^{\mathrm{n}}+\mathrm{F}_{1(2)}^{\mathrm{p}},
$$

where $F_{1(2)}$ denotes the Dirac (Pauli) form factor. As shown in Figure 1 (right), the d-quark contribution seems to scale as $Q^{-4}$ at $Q^{2}>1 \mathrm{GeV}^{2}$, while the u-quark contribution times $Q^{4}$ continually increases in the available data range. Several calculations describe the existing data reasonably well, including the DSE/Faddeev calculation [17] in which the diquark degrees of freedom accounts for the decreased ratio $F_{1}^{d} / F_{1}^{u}$ and $F_{2}^{d} / F_{2}^{u}$ at large $Q^{2}$, and a quark-diquark model with a pion cloud [18], as well as parameterizations of Generalized Parton Distributions (GPDs) [19, 20]. The future experiment [21] will allow the flavor decomposition at $Q^{2}$ up to $10 \mathrm{GeV}^{2}$, where the behaviors of the individual quark contributions as well as the calculations will be further tested.

\subsection{Unified view of nucleon structure}

Together with the Parton Distribution Functions (PDFs), the nucleon form factors have provided important information about the structure of nucleon. While a more comprehensive understanding of the nucleon structures, especially the orbital angular momentum of quarks and gluons, require information from multi-dimensions. An unified view of nucleon structure is possible by introducing the Wigner distributions $W_{p}^{u}\left(x, k_{T}, \vec{r}_{T}\right)$ [22], though its notion contradicts the uncertainty principle that forbids the simultaneous determination of momentum and position. However, by integrating the Wigner distributions over the parton transverse momentum $k_{T}$ or position $r_{T}$, one can obtain the three-dimensional Generalized Parton Distributions (GPDs) or the Transverse Momentum Dependent Parton Distributions (TMDs), which provides key information on the spatial and/or momentum distribution of the partons inside a nucleon. In addition, the PDFs and form factors are also encompassed in the Wigner distributions. Fourier transformations of the nucleon electromagnetic form factors which describe the nucleon's transverse spatial distribution of charge and magnetization can be obtained by integrating the GPDs over Bjorken scaling variable $x$.
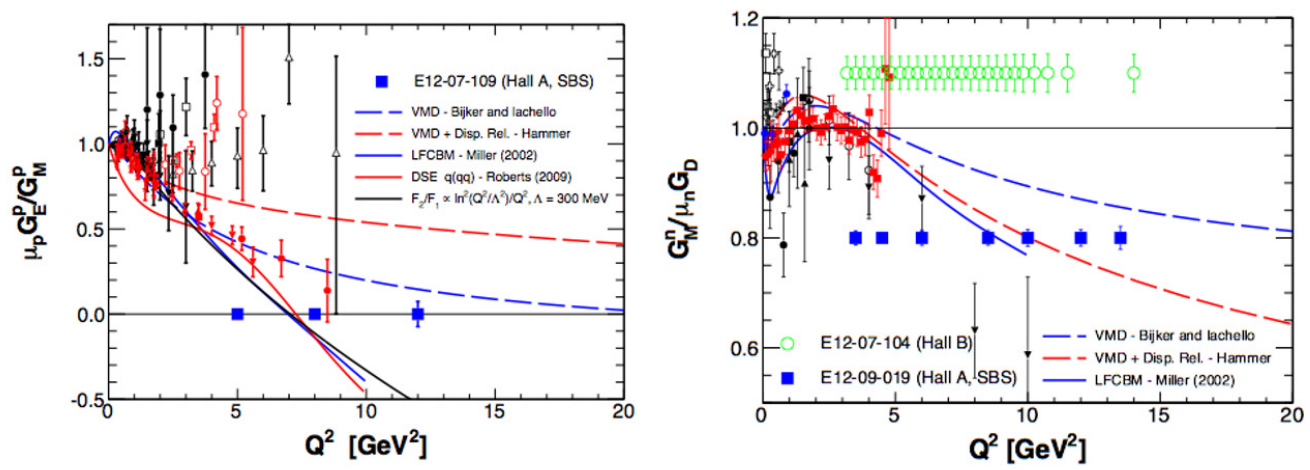

Figure 2. The projections of E12-07-109 [25] and E12-09-019 [26] experiments proposed in Hall A that will utilize the Super Bigbite.

\subsection{Upcoming $12 \mathrm{GeV}$ program at Jefferson Lab}

The Continuous Electron Beam Accelerator Facility (CEBAF) at Jefferson Lab is under an energy upgrade to $12 \mathrm{GeV}$ [23], which is expected to be completed in 2017. The upgraded instrumentation 
enables new experiments that explore the nucleon structures in an unprecedented kinematic coverage. Among those experiments, the Super Bigbite (SBS) projects proposed in Hall A [24] aim to precisely determine the nucleon form factor ratios $G_{E}^{p} / G_{M}^{p}, G_{E}^{n} / G_{M}^{n}$ and $G_{M}^{n} / G_{M}^{p}$ at $Q^{2}$ up to $10-14.5 \mathrm{GeV}^{2}$, which is far greater than the existing data. The Super Bigbite itself refers to a set of components which can be configured to fulfill the requirements of the specified measurement on the form factor $G_{E}^{n}, G_{M}^{n}$ and $G_{E}^{p}$. By utilizing the Gas Electron Multiplier (GEM) technology, all the configurations of SBS have several similar advantages compared with the existing spectrometers: large geometry acceptance, capabilities to detect high rate events and to reach forward angles. Figure 2 shows the projections for two of the proposed SBS experiments.

\section{Proton radius puzzle and PRad experiment}

\subsection{Proton radius puzzle}

In the low $Q^{2}$ region, the "proton radius puzzle" recently triggered major efforts to reevaluate our understanding of the proton charge radius, which was thought as a well known fundamental quantity. Two measurements of the muonic hydrogen Lamb shift at PSI [27, 28] extracted the most precise value on the proton charge radius, but they were $7 \sigma$ smaller than the value of CODATA 2012 compilation [29], which is a weighted average of world data from hydrogen spectroscopic measurements and elastic electron-proton scattering experiments. Figure 3 shows the discrepancy between PSI values and other experiment or analysis results.

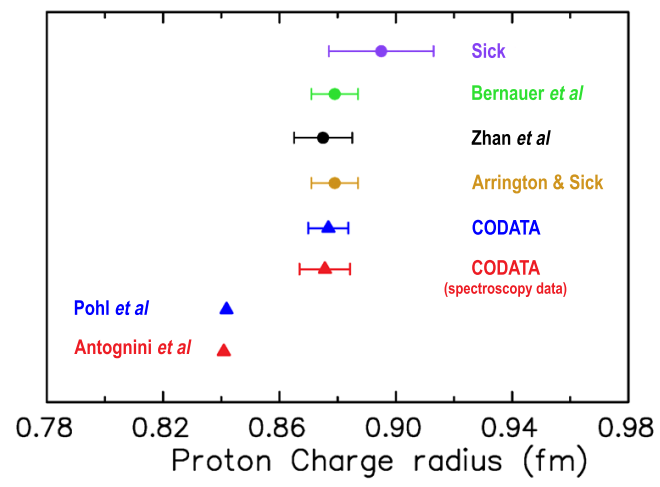

Figure 3. A comparison between the proton charge radius from PSI, CODATA, as well as the elastic ep scattering measurements [30-32] and analyses [33, 34].

This puzzle motivated intensive revisit of the QED calculations of the muonic hydrogen Lamb shift, including the evaluation of the proton polarizability contribution [35-37], yet no terms that can account for this discrepancy were found. The refined calculations were summarized in [38, 39]. New physics that may explain the puzzle was also explored, such as new particles [40, 41], new parity violating muonic force [42], and quantum gravity at the Fermi scale [43], just to name a few. In addition, some explanations in terms of the dispersion relations [44] or frame transformation [45, 46] were proposed to resolve the puzzle, but they remained controversial or unconfirmed. After all, the understanding of the "proton radius puzzle" awaits for input from new dedicated experiments. 


\subsection{Recent proton charge radius measurements}

The absolute frequency of $\mathrm{H}$ energy levels has been measured with an accuracy of 1.4 part in $10^{14}$ via comparison with an atomic cesium fountain clock as a primary frequency standard. Such a precise measurement with the state-of-the-art QED calculations that include corrections for the finite size of the proton can indirectly determine the proton charge radius. The precision of this method is significantly enhanced by measuring the Lamb shift of muonic hydrogen. The contribution from the proton finite size term to the Lamb shift is much larger in muonic hydrogen than that in atomic hydrogen due to the fact that the muon mass is about 206 times of the electron mass. Such measurement was achieved at PSI in 2010 [27], and its result was reinforced by the same group in 2013 [28], which reported that $r_{p}=0.84087 \pm 0.00039$. These measurements obtained the most precise value of the proton charge radius; its uncertainty was less than $0.05 \%$. However, the PSI value is $4 \%$ less than the CODATA value, as well as the results from the recent $e p$ scattering measurements.

Alternatively, the proton charge radius can be directly determined based on the elastic ep scattering experiments. Since the proton electric form factor $G_{E}^{p}$ can be interpreted as the Fourier transform of the spatial charge distribution, the proton charge radius is extracted by fitting the slope of $G_{E}^{p}\left(Q^{2}\right)$ to $Q^{2} \rightarrow 0$ :

$$
\left\langle r^{2}\right\rangle=-\left.6 \frac{d G_{E}^{p}\left(Q^{2}\right)}{d Q^{2}}\right|_{Q^{2}=0} .
$$

Thus, the $G_{E}^{p}$ data at low $Q^{2}$ are essential for this method.

The recent experiment at Mainz [30,31] measured the cross sections of elastic ep scattering within $Q^{2}=0.003-1.0 \mathrm{GeV}^{2}$. Three spectrometers were utilized to cover a large amount of overlapping data sets. The statistical uncertainties of the measured cross-sections are lower than $0.2 \%$. Both the electric and magnetic form factors of the proton were extracted from fitting the cross-section data. The recoil proton polarization experiment at Jefferson Lab [32] measured the elastic form factor ratio $\mu_{p} G_{E} / G_{M}$ of proton for $Q^{2}=0.3-0.7 \mathrm{GeV}^{2}$. They extracted the proton charge radius by fitting the global data at $Q^{2}<0.5 \mathrm{GeV}^{2}$. The two experimental results are consistent with each other. However, they are $3-5 \sigma$ larger than the PSI value.

\subsection{PRad experiment at Jefferson Lab}

To investigate the proton charge radius puzzle, a precise experiment based on the ep elastic scattering with totally different systematic errors is necessary to check the result from previous ep scattering experiments. Thus, the proton charge radius (PRad) experiment is approved with A rating in Hall B, at Jefferson Lab.

The PRad experiment aims to precisely determine the proton charge radius based on the crosssections measurement of elastic ep scattering with a calorimetry method. As shown in Figure 4, the experiment utilizes a high resolution, high efficiency calorimeter ( $\mathrm{HyCal}$ ) as the main detector. The calorimetric setup enables the experiment to reach the very forward angles and thus the very low $Q^{2}$ region $\left(\geq 2 \times 10^{-4} \mathrm{GeV}^{2}\right)$ that was never achieved before by magnetic spectrometer based measurements. As supplemental detectors, two GEM chambers at the upstream of HyCal can significantly increase the position resolution and reduce the systematic errors in the $Q^{2}$ determination. The hydrogen target used in PRad is a windowless gas-flow target. By removing the target windows, this experiment will not suffer from the typical background source of all previous $e p$ experiments in order to reach the most forward scattering angles. The scattered electron from the elastic ep scattering and Møller scattering will be simultaneously detected. The cross-sections of elastic ep scattering hence will be normalized to those of the Møller process, which can be precisely calculated in QED. 


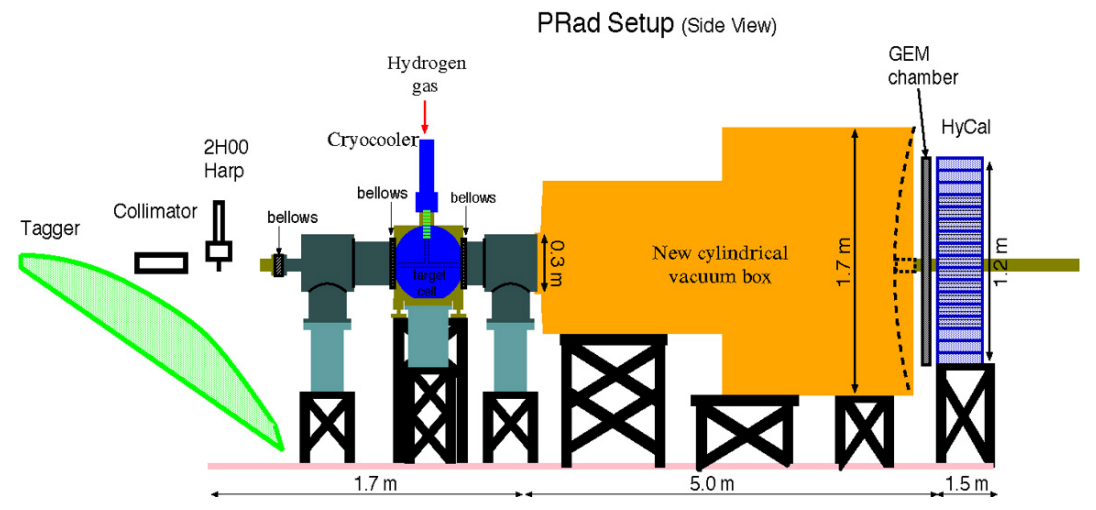

Figure 4. The layout of the PRad experiment, it is not to scale.

The extracted proton charge radius is expected to have a sub-percent precision. The systematic errors will be totally different compared with previous spectrometer based experiments. Its result will evaluate the discrepancy between the $e p$ scattering experiments and the Lamb shift measurements of muonic hydrogen. Together with the $\mu p$ elastic scattering experiment at PSI [47] and the Initial State Radiation (ISR) experiments at Mainz [48], these high precision experiments will have direct impacts on the "proton radius puzzle".

\section{Summary}

The nucleon electromagnetic form factors remain at the forefront of studies of the nucleon structure. On one hand, the 12-GeV CEBAF enables experiments that explore nucleon form factors at higher momentum transfers squared. On the other hand, the "proton radius puzzle", which is related to the proton electric form factor at low momentum transfers squared, remains unresolved, and awaits new experimental results.

\section{Acknowledgements}

This work is supported in part by U.S. Department of Energy under contract number DE-FG02-03ER41231 and NSF MRI award PHY-1229153. We thank J. Arrington, G. Cates, D. Dutta, R. Ent, A. Gasparian, R. Holt, M. Khandaker, M. Meziane, J.W. Qiu, and C. Roberts for useful discussions.

\section{References}

[1] C. F. Perdrisat, V. Punjabi, M. Vanderhaeghen, Prog. Part. Nucl. Phys. 59, 694 (2007).

[2] G. D. Cates et al., Phys. Rev. Lett. 106, 252003 (2011).

[3] C. Carlson, Prog. Part. Nucl. Phys. 82, 59 (2015).

[4] M. N. Rosenbluth, Phys. Rev. 79, 615 (1950).

[5] T. W. Donnelly and A. S. Raskin, Ann. Phys. 169, 247 (1986).

[6] C. Crawford, Phys. Rev. Lett. 98, 052301 (2007).

[7] S. J. Brodsky and G. R. Farrar, Phys. Rev. D 11, 1309 (1975). 
[8] G. P. Lepage and S. J. Brodsky, Phys. Rev. D 22, 2157 (1980).

[9] A. J. R. Puckett et al., Phys. Rev. C 85, 045203 (2012).

[10] A. V. Belitsky, X. Ji and F. Yuan, Phys. Rev. Lett. 91, 092003 (2003).

[11] G. A. Miller and M. R. Frank, Phys. Rev. C 65, 065205 (2002).

[12] S. Boffi et al., Eur. Phys. J. A 14, 17 (2002).

[13] F. Cardarelli el al., Phys. Lett. B 357, 267 (1995).

[14] F. Cardarelli and S. Simula, Phys. Rev. C 62, 65201 (2000).

[15] P. L. Chung and F. Coester, Phys. Rev. D 44, 229 (1991).

[16] F. Gross and P. Agbakpe, Phys. Rev. C 73, 015203 (2006).

[17] I. C. Cloët et al., Few Body Syst. 46, 1 (2009).

[18] I. C. Cloët and G. A. Miller, Phys. Rev. C 86, 015208 (2012).

[19] M. Guidal et al., Phys. Rev. D 72, 054013 (2005).

[20] M. Diehl and P. Kroll, Eur. Phys. J. C 73, 2397 (2013).

[21] G. Cates, S. Riordan, and W. Bogdan (spokespersons), Experiment E12-09-016 at Jefferson Lab.

[22] X. Ji, Phys. Rev. Lett. 91, 062001 (2003).

[23] Dudek et al., arXiv:1208.1244 [hep-ex] (2012); https://www.jlab.org/12GeV.

[24] K. de Jager Int. J. Mod. Phys. E 19, 844 (2010).

[25] L. P. Pentchev (contact person) et al., Experiment E12-07-109 at Jefferson Lab.

[26] B. Wojtsekhowski (contact person) et al., Experiment E12-09-019 at Jefferson Lab.

[27] R. Pohl et al., Nature 466, 213 (2010).

[28] A. Antognini et al., Science 339, 417 (2013).

[29] P.J. Mohr, B.N. Taylor, and D.B. Newell, Rev. Mod. Phys. 84, 1527 (2012).

[30] J.C. Bernauer et al., Phys. Rev. Lett. 105, 242001 (2010).

[31] J. Bernauer et al., Phys. Rev. C 90, 015206 (2014).

[32] X. Zhan et al., Phys. Rev. B 705, 59 (2011).

[33] I. Sick, Phys. Lett. B 576, 62 (2003).

[34] J. Arrington and I. Sick, J. Phys. Chem. Ref. Data 44, 031204 (2015).

[35] M. C. Birse and J. A. McGovern et al., Eur. Phys. J. A 48, 120 (2012).

[36] G. A. Miller, Phys. Lett. B 718, 1078 (2013).

[37] J. M. Alarcón, V. Lensky, and V. Pascalutsa, Eur. Phys. J. C 74, 2852 (2014).

[38] U.D. Jentschura, Ann. Phys. 326, 500 (2011).

[39] A. Antognini et al., Ann. Phys. 331, 127 (2013).

[40] V. Barger et al., Phys. Rev. Lett. 106, 153001 (2011).

[41] C. Carlson and B. Rislow, Phys. Rev. D 86, 035013 (2012).

[42] B. Batell, D. Mckeen and M. Pospelov Phys. Rev. Lett. 107, 011803 (2011).

[43] R. Onofrio, Europhys. Lett. 104, 20002 (2013).

[44] I. T. Lorentz, H.-W. Hammer, Ulf-G. Meißner, Eur. Phys. J. A 48, 151 (2012).

[45] D. Robson Int. J. Mod. Phys. E 23, 14500906 (2014).

[46] T. W. Donnelly, D. K. Hasell, and R. G. Milner, arXiv:1505.04723 [nucl-ex] (2015).

[47] R. Gilman et al., arXiv:1303:2160v1 [nucl-ex] (2013).

[48] M. Mihovilovic et al., EPJ Web Conf. 72, 00017 (2014). 